\title{
Synchronously Pumped Optical Parametric Oscillator With a Repetition Rate of $81.8 \mathrm{GHz}$
}

\author{
Steve Lecomte, Rüdiger Paschotta, Susanne Pawlik, Berthold Schmidt, Kentaro Furusawa, Andrew Malinowski, \\ David J. Richardson, and Ursula Keller, Senior Member, IEEE
}

\begin{abstract}
We present a singly resonant synchronously pumped optical parametric oscillator with a record-high pulse repetition rate of $81.8 \mathrm{GHz}$. It generates up to $0.9 \mathrm{~W}$ of signal average output power at a wavelength of $1569.7 \mathrm{~nm}$. The pulses have a duration of $2.4 \mathrm{ps}$ and are nearly transform-limited. The device is wavelength tunable from 1541.4 to $1592.2 \mathrm{~nm}$. The tuning range could be easily extended by the use of a multiperiod periodically poled $\mathrm{LiNbO}_{3}$ crystal.
\end{abstract}

Index Terms-High pulse repetition rates, mode-locked lasers, optical fiber amplifiers, optical parametric oscillators (OPOs).

\section{INTRODUCTION}

$\mathbf{O}$ PTICAL pulse sources with multigigahertz repetition rates are of great importance for actual telecommunication networks. Future return-to-zero formats will take advantage of directly pulsed sources. Several approaches have been explored to generate pulse trains with very high repetition rates: harmonically mode-locked fiber ring lasers, hybrid mode-locked semiconductor lasers, and passively mode-locked solid-state lasers. For test and measurement applications, pulsed sources should ideally be widely wavelength tunable and provide a high average output power in short pulses. Particularly in this domain, the 81.8-GHz parametric oscillator described in this letter appears to be an interesting source.

We first consider some competing technologies. Harmonically mode-locked fiber-ring lasers are a standard approach to generate picosecond pulses at gigahertz pulse repetition rates. Actively mode-locked lasers with up to $80 \mathrm{GHz}$ [1] have been demonstrated with harmonic mode locking. Higher repetition rates were achieved with pulse repetition rate multiplication or rational harmonic mode-locking [2]. However, this kind of source is limited in terms of average output powers to a few milliwatts. Wavelength tuning can in general be performed over some tens of nanometers, covering, e.g., the telecommunication $C$ band [3]. A major drawback is that fiber lasers require sophisticated stabilization schemes for stable operation.

Manuscript received August 25, 2004; revised September 23, 2004. This work was supported in part by the Swiss innovation promotion agency KTI/CTI (Commission for Technology and Innovation) and by Hasler Stiftung.

S. Lecomte, R. Paschotta, and U. Keller are with the Physics Department, Institute of Quantum Electronics, ETH Zürich CH-8093, Switzerland (e-mail: lecomte@phys.ethz.ch; paschotta@phys.ethz.ch; keller@phys.ethz.ch).

S. Pawlik and B. Schmidt are with Bookham (Switzerland) AG, Zürich CH-8045, Switzerland (e-mail: susanne.pawlik@bookham.com; berthold.schmidt@bookham.com).

K. Furusawa, A. Malinowski, and D. J. Richardson are with the Optoelectronics Research Center (ORC), University of Southampton, Southampton SO17 1BJ, U.K. (e-mail: anm@ orc.soton.ac.uk; djr@orc.soton.ac.uk).

Digital Object Identifier 10.1109/LPT.2004.839385
Actively and passively mode-locked edge-emitting semiconductor lasers have been used for high pulse repetition rates of up to $1.2 \mathrm{THz}$ [4], but with rather limited average output power and wavelength tunability.

Passively mode-locked solid-state lasers attracted a lot of attention in recent years. $\mathrm{Nd}: \mathrm{YVO}_{4}$ lasers emitting at $1.064 \mu \mathrm{m}$ were demonstrated with repetition rates of up to $\approx 160 \mathrm{GHz}$, and a 10-GHz diode-pumped laser generated up to $2.1 \mathrm{~W}$ of average output power [5]. Similar lasers emitting at telecom wavelengths and based on $\mathrm{Er}: \mathrm{Yb}$ : glass as gain medium were demonstrated at repetition rates of up to $50 \mathrm{GHz}$ [6]. So far, devices with repetition rates of up to $25 \mathrm{GHz}$ could be tuned over the whole $C$ band [7]. Recently, a novel kind of semiconductor laser, the passively mode-locked vertical extended cavity semiconductor laser, has shown to have the potential of achieving high repetition rates in combination with high average powers. A 10-GHz device emitting $1.4 \mathrm{~W}$ of average output power in 6.1-ps pulses at a central wavelength of $960 \mathrm{~nm}$ [8] has been demonstrated.

Here we present a less common approach based on a singly resonant synchronously pumped optical parametric oscillator (OPO) which is pumped with an amplified diode-pumped passively mode-locked $\mathrm{Nd}: \mathrm{YVO}_{4}$ miniature laser. This approach has highly desirable properties, in particular a very high average output power and an ultrabroad wavelength tuning range, thus outperforming all the other types of multigigahertz sources emitting in the $1.5-\mu \mathrm{m}$ spectral region. We previously reported about similar devices at lower repetition rates. A 10-GHz OPO could be tuned over $154 \mathrm{~nm}$ covering the $S, C$, and $L$ bands [9], whereas a $39-\mathrm{GHz}$ OPO generated up to $2.1 \mathrm{~W}$ of signal average output power [10]. In this letter, we present a record-high repetition rate of $81.8 \mathrm{GHz}$ from a similar $\mathrm{OPO}$ which generates up to $0.9 \mathrm{~W}$ of average output power at $1569.7 \mathrm{~nm}$. Moreover, the $\mathrm{Nd}: \mathrm{YVO}_{4}$ seed laser used here has the highest repetition rate demonstrated so far for a diode-pumped passively mode-locked solid-state laser.

\section{EXPERIMENTAL SETUP}

The 81.8-GHz repetition rate seed laser is a diode-pumped quasi-monolithic $\mathrm{Nd}: \mathrm{YVO}_{4}$ laser [5] which is passively mode-locked with a semiconductor saturable absorber mirror (SESAM) [11]. This laser is similar to one of the two lasers described in [12] except that the crystal radius of curvature is $5 \mathrm{~mm}$ instead of $10 \mathrm{~mm}$ and that the crystal thickness is $0.82 \mathrm{~mm}$ instead of $1.74 \mathrm{~mm}$, corresponding to a fundamental repetition rate of $81.8 \mathrm{GHz}$. It delivers transform-limited 3.3-ps pulses with an average output power of $100 \mathrm{~mW}$ at a wavelength of $1.064 \mu \mathrm{m}$. 
The main challenge in operating a multigigahertz OPO is to overcome the pump threshold. Because state-of-the-art passively mode-locked $\mathrm{Nd}: \mathrm{YVO}_{4}$ lasers [12] do not provide sufficient power, we used a seed laser optimized for short pulses, the output of which we subsequently amplify with an efficient Yb-doped fiber amplifier. The fiber amplifier was end-pumped with a fiber-coupled $24-\mathrm{W} 915$-nm diode. The $\approx 4.5$-m-long step-index $\mathrm{Yb}$-doped large mode area fiber has a core diameter of $30 \mu \mathrm{m}(\mathrm{NA}=0.06)$ and a D-shaped inner cladding with a diameter of $300 \mu \mathrm{m}$. The core was doped with $8000 \mathrm{ppm}$ of $\mathrm{Yb}^{3+}$ ions. An amplifier for high energy femtosecond pulses from a fiber oscillator [13] and a $Q$-switched fiber laser [14] were recently demonstrated using fibers of the same design. The duration and spectral width of the output pulses are unchanged during the amplification, as confirmed by experiments and calculations. The amplifier was saturated with a few milliwatts of coupled seed power and produced up to $9.8 \mathrm{~W}$ of average power. The beam was diffraction-limited $\left(M^{2}<1.1\right)$ and linearly polarized, as required for pumping the OPO, after a polarization controller placed before the amplifier.

The OPO has a 21-mm-long periodically poled $\mathrm{LiNbO}_{3}$ (PPLN) crystal from Crystal Technology Inc., which is antireflection-coated for the pump, signal, and idler wavelengths. The poled grating period is $29.6 \mu \mathrm{m}$ and the crystal is kept at $180^{\circ} \mathrm{C}$ in a homemade temperature-stabilized oven. To minimize the intracavity signal losses and to efficiently damp the idler wave, we use a singly resonant ring cavity [15]. The two curved cavity mirrors have a radius of curvature of $75 \mathrm{~mm}$ and are $96 \mathrm{~mm}$ apart, whereas, the two other mirrors are flat. The second curved mirror is the output coupler and has a transmission of $0.22 \%$ at the signal wavelength. The free-spectral range of the cavity is $629 \mathrm{MHz}$, corresponding to 130 pulses simultaneously circulating in the cavity, when pumped with a repetition rate of $81.8 \mathrm{GHz}$. Although this situation is reminiscent of harmonic mode locking, it does not introduce problems with supermodes [16] or timing jitter, as the timing of all circulating pulses is determined by the timing of the pump pulses. The advantage of a subharmonic cavity is to use a long nonlinear crystal to maximize the OPO gain and, therefore, to minimize the OPO threshold. The signal waist's radius in the PPLN crystal is $49.6 \mu \mathrm{m}$, resulting in a focusing parameter $\xi_{s}$ (ratio of the crystal length to the confocal parameter in the crystal) of 0.99 . The pump beam is focused to a radius of $43 \mu \mathrm{m}$, corresponding to a focusing parameter $\xi_{p}$ of 0.90 . We conservatively choose the signal and pump radii to avoid damage of the PPLN crystal. Fig. 1 shows a schematic of the experimental setup.

\section{RESULTS}

With 6.1 W of available pump power incident on the PPLN crystal, $0.9 \mathrm{~W}$ of signal average power at a wavelength of $1569.7 \mathrm{~nm}$ is generated. The pulse length is $2.4 \mathrm{ps}$ and the pulses are almost transform-limited. Fig. 2 shows the autocorrelation trace and the optical spectrum. The pump depletion reached $60 \%$ at full pump power. The signal round-trip losses were calculated by using the Manley-Rowe relation (corresponding to the photon-number conservation) to be $0.4 \%$.

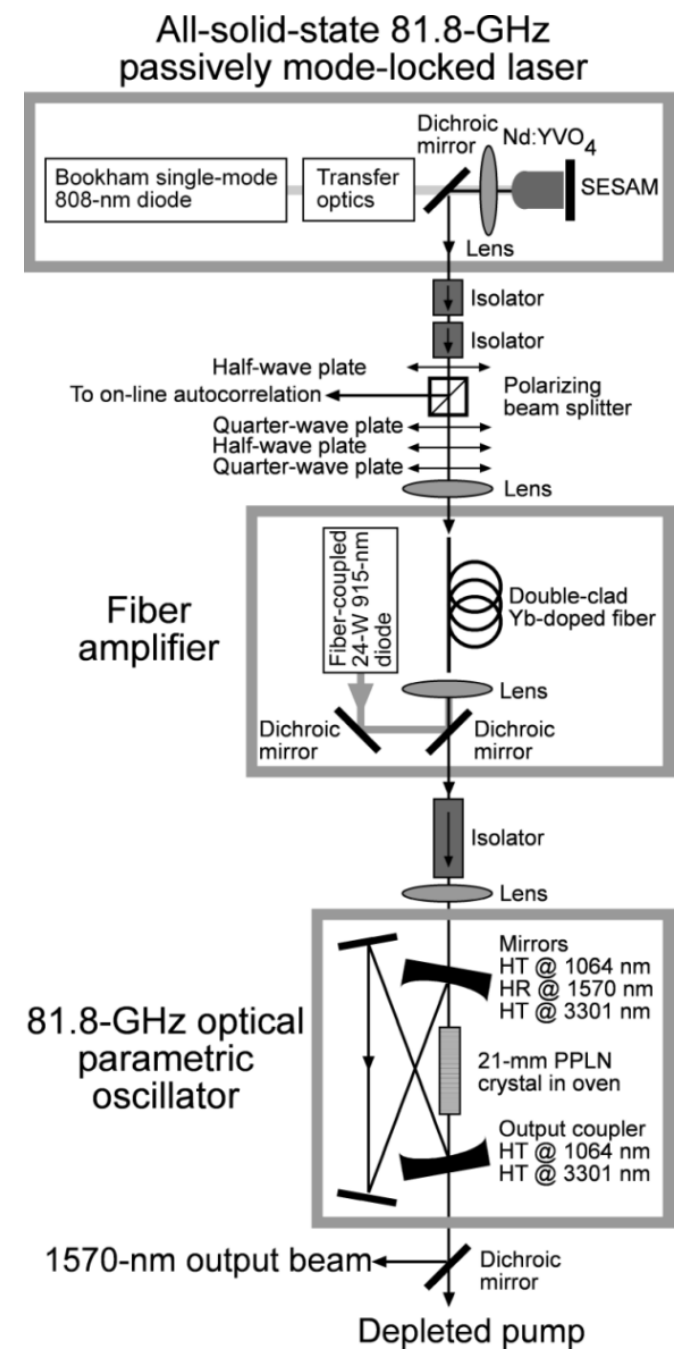

Fig. 1. Experimental setup. 81.8-GHz diode-pumped passively mode-locked $\mathrm{Nd}: \mathrm{YVO}_{4}$ laser, $\mathrm{Yb}$-doped fiber amplifier, and OPO. HT: High transmission. HR: High reflectivity.
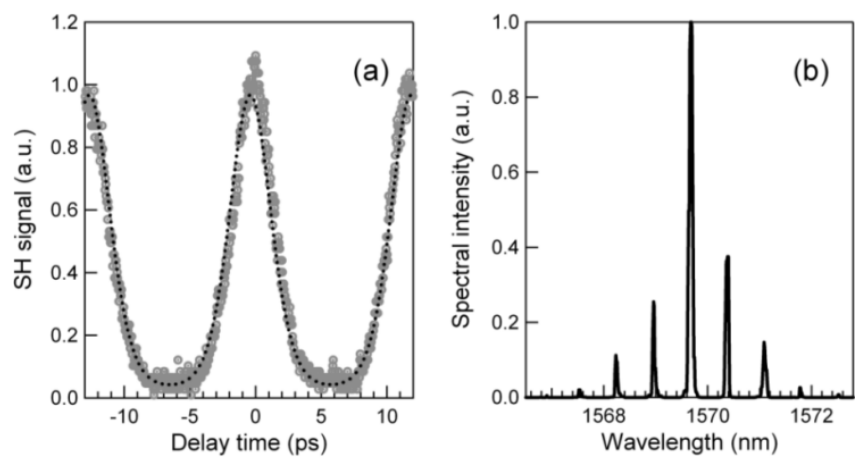

Fig. 2. (a) Measured autocorrelation (circles) of the signal pulses with 0.9-W average power. The pulse length is $2.4 \mathrm{ps}$, assuming a sech ${ }^{2}$ shape (fit: dotted line). (b) Optical spectrum of the $81.8-\mathrm{GHz}$ pulse train with $0.9-\mathrm{W}$ average power taken with $0.08-\mathrm{nm}$ resolution. The longitudinal modes with $81.8-\mathrm{GHz}$ spacing are resolved.

With full pump power, the OPO oscillates for a cavity detuning range of several millimeters. In fact, we reached enough pump spectral intensity to overcome the continuous-wave (CW) 
OPO oscillation threshold. The OPO produced up to 400-mW $\mathrm{CW}$ average signal power. When the OPO was synchronously pumped, it generated pulses on a cavity detuning range of $25 \mu \mathrm{m}$. For long-term stable operation, a feedback stabilization of the relative cavity lengths would be required.

By varying the PPLN crystal temperature from $120{ }^{\circ} \mathrm{C}$ to $220^{\circ} \mathrm{C}$, we tuned the signal wavelength from 1541.4 to $1592.2 \mathrm{~nm}$. The idler wavelength varies from 3435 to $3207 \mathrm{~nm}$ but was not measured because the mirror substrates were made of BK7 glass which is strongly absorbing above a wavelength of $2.8 \mu \mathrm{m}$. As demonstrated with a $10-\mathrm{GHz}$ OPO [9], the wavelength tuning could be easily further extended by the use of a multiperiod PPLN crystal covering the $S, C$, and $L$ bands for telecom applications.

\section{CONCLUSION}

We have presented a singly resonant synchronously pumped OPO with a record-high repetition rate of $81.8 \mathrm{GHz}$. The device produced up to $0.9 \mathrm{~W}$ of signal average output power in almost transform-limited pulses at $1569.7 \mathrm{~nm}$. Since enough pump power is available to reach the OPO threshold even in $\mathrm{CW}$ operation, the OPO operates also when its cavity length is not matched to that of the pump laser, then generating CW output. The combination of high repetition rate, high average output power, and broad tuning range is by far superior to that of any other demonstrated pulse source in the $1.5-\mu \mathrm{m}$ region.

Even higher average output powers from the OPO should be feasible by increasing the pump power of the fiber amplifier. Higher repetition rates also appear to be feasible, if a suitable seed laser is available. The latter is challenging, however, as passively mode-locked $\mathrm{Nd}: \mathrm{YVO}_{4}$ lasers are limited in terms of short pulse duration to $\approx 2.7 \mathrm{ps}$ [5], and shorter pump pulses are required for achieving repetition rates well above $100 \mathrm{GHz}$ with well separated pulses. An option is to spectrally broaden the pump pulses in a fiber and recompress them with appropriate dispersion.

\section{ACKNOWLEDGMENT}

The authors would like to thank GigaTera Inc. as industrial partner, and L. Krainer and G. J. Spühler for fruitful discussions.

\section{REFERENCES}

[1] L. Schares, L. Occhi, and G. Guekos, "80-160 GHz mode-locked fiber ring laser synchronized to external optical pulse stream," IEEE Photon. Technol. Lett., vol. 15, no. 10, pp. 1348-1350, Oct. 2003.

[2] E. Yoshida and M. Nakazawa, "80-200 GHz erbium doped fiber laser using a rational harmonic mode-locking technique," Electron. Lett., vol. 32, pp. 1370-1372, 1996.

[3] B. Bakhshi and P. A. Andrekson, "40-GHz actively modelocked polarization-maintaining erbium fiber ring laser," Electron. Lett., vol. 36, pp. 411-412, 2000.

[4] S. Arahira, Y. Matsui, and Y. Ogawa, "Mode-locking at very high repetition rates more than terahertz in passively mode-locked distributedBragg-reflector laser diodes," IEEE J. Quantum Electron., vol. 32, no. 7, pp. 1211-1224, Jul. 1996.

[5] L. Krainer, R. Paschotta, S. Lecomte, M. Moser, K. J. Weingarten, and U. Keller, "Compact $\mathrm{Nd}: \mathrm{YVO}_{4}$ lasers with pulse repetition rates up to 160 GHz,” IEEE J. Quantum Electron., vol. 38, no. 10, pp. 1331-1338, Oct. 2002.

[6] S. C. Zeller, L. Krainer, G. J. Spühler, R. Paschotta, M. Golling, D. Ebling, K. J. Weingarten, and U. Keller, "Passively mode-locked 50-GHz Er: Yb:glass laser," Electron. Lett., vol. 40, pp. 875-876, 2004.

[7] G. J. Spühler, P. S. Golding, L. Krainer, I. J. Kilburn, P. A. Crosby, M. Brownell, K. J. Weingarten, R. Paschotta, M. Haiml, R. Grange, and U. Keller, "Novel multi-wavelength source with $25-\mathrm{GHz}$ channel spacing tunable over the C-band," Electron. Lett., vol. 39, pp. 778-780, 2003.

[8] A. Aschwanden, D. Lorenser, H. J. Unold, R. Paschotta, E. Gini, and U. Keller, "10-GHz passively mode-locked surface-emitting semiconductor laser with 1.4 W output power," in Conf. Lasers and Electro-Optics (CLEO) (Optical Society of America), San Francisco, CA, 2004, Postdeadline Paper CPDB8.

[9] S. Lecomte, R. Paschotta, M. Golling, D. Ebling, and U. Keller, "Synchronously pumped optical parametric oscillators in the $1.5-\mu \mathrm{m}$ spectral region with a repetition rate of $10 \mathrm{GHz}$, , J. Opt. Soc. Amer. B, vol. 21, pp. 844-850, 2004.

[10] S. Lecomte, R. Paschotta, S. Pawlik, B. Schmidt, K. Furusawa, A. Malinowski, D. J. Richardson, and U. Keller, "39-GHz repetition rate optical parametric oscillator in the $1.5-\mu \mathrm{m}$ spectral region," Opt. Lett., to be published.

[11] U. Keller, K. J. Weingarten, F. X. Kärtner, D. Kopf, B. Braun, I. D. Jung, R. Fluck, C. Hönninger, N. Matuschek, and J. A. der Au, "Semiconductor saturable absorber mirrors (SESAM's) for femtosecond to nanosecond pulse generation in solid-state lasers," IEEE J. Sel. Topics Quantum Electron., vol. 2, no. 3, pp. 435-453, Sep. 1996.

[12] S. Lecomte, M. Kalisch, L. Krainer, G. J. Spühler, R. Paschotta, L. Krainer, M. Golling, D. Ebling, T. Ohgoh, T. Hayakawa, S. Pawlik, B. Schmidt, and U. Keller, "Diode-pumped passively mode-locked $\mathrm{Nd}: \mathrm{YVO}_{4}$ lasers with $40-\mathrm{GHz}$ repetition rate," IEEE J. Quantum Electron., to be published.

[13] A. Malinowski, A. Piper, J. H. V. Price, K. Furusawa, Y. Jeong, J. Nilsson, and D. J. Richardson, "Ultra-short pulse $\mathrm{Yb}^{3+}$-fiber-based laser and amplifier system producing $>25-\mathrm{W}$ average power," Opt. Lett., vol. 29, pp. 2073-2075, 2004.

[14] A. Piper, A. Malinowski, K. Furusawa, and D. J. Richardson, "High-power, high-brightness, mJ Q-switched ytterbium doped fiber laser," Electron. Lett., vol. 40, pp. 928-929, 2004.

[15] W. R. Bosenberg, A. Drobshoff, J. I. Alexander, L. E. Myers, and R. L. Byer, "93\% pump depletion, 3.5-W continuous-wave, singly resonan optical parametric oscillator," Opt. Lett., vol. 21, pp. 1336-1338, 1996.

[16] M. F. Becker, K. J. Kuizenga, and A. E. Siegman, "Harmonic mode locking of the Nd: YAG laser," IEEE J. Quantum Electron., vol. QE-8, no. 8, pp. 687-693, Aug. 1972. 